\title{
Nephrostomy in pregnancy a district general hospital experience over five years
}

\author{
Anthony Kodzo-Grey Venyo*1, Christiana Oluwashola Fatola ${ }^{2}$, Dayo Adegbite ${ }^{3}$, Ali Nawaz Khan ${ }^{4}$ \\ ${ }^{1}$ Department of Urology, North Manchester General Hospital, Manchester, United Kingdom \\ ${ }^{2}$ Department of Ear Nose and Throat, North Manchester General Hospital, Manchester, United Kingdom \\ ${ }^{3}$ Department of Obstetrics and Gynaecology, North Manchester General Hospital, Manchester, United Kingdom \\ ${ }^{4}$ Department of Radiology, North Manchester General Hospital, Manchester, United Kingdom
}

Received: June 19, 2014

DOI: $10.5430 / j b g c . v 5 n 1 p 1$
Accepted: September 9, 2014 Online Published: November 30, 2014

URL: http://dx.doi.org/10.5430/jbgc.v5n1p1

\begin{abstract}
Background: Obstructed kidney is also termed hydronephrosis. An obstructed kidney produces buildup of urine within the kidney which increases pressure within the kidney. This condition can be a result of several different causes of which pregnancy is one of them. Obstructed kidneys in pregnancy call for relieving by nephrostomy which is a thin, plastic tube (catheter) that is inserted through the skin on the back and into the kidney. It can relieve a build-up of urine in the kidney and prevents the kidney from being damaged. It drains urine directly from one or both kidneys into a collecting bag outside the body. The bag has a tap so it can be emptied.

Aims: To find out the rate of nephrostomy tube insertion in pregnant women in North Manchester General Hospital between January 2009 - December 2013. To identify problems that emanated from insertion of the nephrostomies.

Results: Fifteen patients out of 24,863 deliveries $(0.06 \%)$ in our catchment area underwent 17 nephrostomy procedures. All the nephrostomy insertions were successfully and safely undertaken. There was no immediate complication. The complications that were noted include: subsequent blockage of nephrostomy tubes which required readmission to flush the tubes to render them patent, two nephrostomy tubes subsequently fell out which were replaced, there was 1 minor infection which was treated by oral antibiotics. Surgical causes of obstruction found at post-partum nephrostogram include calculi and ureteric strictures which were managed post-partum. All the pregnancies continued to full-term with delivery of normal full-term babies.

Conclusions: Percutaneous nephrostomy insertion in the pregnant woman under ultra-sound scan guidance is a safe and effective procedure associated with minimal complications and this allows pregnancy to continue to full term. The fact that the blocked nephostomies could all be flushed easily to unblock the tubes would indicate that if all district nurses are taught how to carefully flush nephrostomies readmission with blocked nephrostomies would be minimised. Strict adherence to nephrostomy protocol would partly help reduce complications associated with nephrostomy insertion in pregnancy.
\end{abstract}

Key Words: Nephrostomy in pregnancy, Calculus, Ureteric stricture, Ultra-sound scan, Nephrostogram

\section{Introduction}

Hydro-ureter and hydronephrosis are physiological changes which often occur in pregnancy. The process of hy- droureteronephrosis may start as early as the sixth week of pregnancy and $90 \%$ of pregnant women would have some element of hydronephrosis by the 28th week of gestation. ${ }^{[1-3]}$

\footnotetext{
*Correspondence: Anthony Kodzo-Grey Venyo; Email: akodzogrey@yahoo.co.uk; Address: Department of Urology, North Manchester General Hospital, Delaunays Road, Manchester, United Kingdom. 
The mechanism of the development of hydro-ureter and hydronephrosis during pregnancy is not very clear; nevertheless, it may be a sequel of a combination pressure on the ureters at the pelvic brim and smooth muscle relaxation pursuant to the hormonal changes associated with pregnancy. Hydro-ureter and hydro-nephrosis of pregnancy is more common on the right side, perhaps because of dextrorotation of the uterus and the sigmoid colon cushioning the ureter on the left. ${ }^{[1,2,4]}$

In majority of pregnancies these changes of hydroureteronephrosis within the upper urinary tract remain asymptomatic and do not emanate in complications. ${ }^{[2]}$ The aforementioned changes have therefore been referred to as physiological changes. ${ }^{[2]}$

It had been stated that five per-cent to ten per-cent of pregnant women develop flank pain necessitating investigations and referral for urological opinion. ${ }^{[1,2]} \mathrm{Hy}-$ dronephrosis leads to urinary stasis which predisposes the patient to develop urinary tract infection and infected hyroureteronephrosis, pyelonephritis, as a complication of bacteriuria, and urolithiasis. ${ }^{[2,5,6]}$ Pyonephrosis may also develop.

The aforementioned complications have been associated with spontaneous abortion, hypertension, pre-term labour, and low birth weight. ${ }^{[6,7]}$ It could be difficult clinically to establish a diagnosis of pathological obstruction in a symptomatic pregnant woman with loin pain and hydronephrosis, especially due to ureteric calculi as the symptoms of nausea, vomiting and back/loin pain, urinary frequency and dysuria/painful micturition could all usually be present during the process of a normal pregnancy. ${ }^{[2]}$ Furthermore, it is difficult to image the ureters in pregnancy, as computed tomography (CT) scan is generally avoided, ${ }^{[2]}$ in order to avoid radiation to the foetus.

Percutaneous nephrostomy (PCN) insertion is one of the common initial treatment options to alleviate painful hydronephrosis during pregnancy temporarily and to maintain renal function as well as to allow pregnancy to continue to full term in order to enable investigation to establish finally the actual cause of the hydronephrosis after the baby has been delivered and to treat any surgical causes found postpartum. By adopting this approach radiation to the foetus is avoided. Presence of nephrostomy in situ for a number of weeks or months before delivery may be inconvenient for the pregnant woman and some expecting mothers have difficulty coping with nephrostomy tubes. Furthermore, there may be nephrostomy associated problems that occur continuously or intermittently which must be dealt with.

The ensuing paper has narrated 5-years audit experience managing nephrostomy in pregnancy in a district general hospital with a discussion of other ways of managing painful hydronephrosis in pregnancy.

\section{Methods}

\subsection{Percutanous nephrostomy insertion techniques}

Two different ultrasound-guided techniques can be used for the insertion of percutaneous nephrostomy including: the Seldinger technique and the "one-step" technique. ${ }^{[1]}$ The patients are placed in prone or in prone-oblique position for the procedure. The patients are given antibiotics routinely prophylactically preceding the procedure. Analgesia is given (for example Pethidine intramuscularly or by Intravenous titration method) as well as an anti-emetic, Coagulation screen is also done prior to the procedure which is usually carried out if the INR is 1.3 or below.

The selection criteria for each technique, depends upon the anatomical configuration of the renal collecting system. The "Seldinger technique" is the method of choice for non-dilated collecting systems and cases with suspected pyonephrosis. This technique is performed with ultrasound scan and fluoroscopic guidance. The "one step" (Bonanno) technique is used without fluoroscopic guidance for moderate-to-severe dilated collecting systems.

The "Seldinger" technique involves ultrasound-guided puncture of the dilated renal collecting system with a 19G sheathed needle, the insertion of a 0.0038 ' heavy-duty $\mathrm{J}$ guide-wire and serial dilatation of the tract with 6 to $10 \mathrm{~F}$ dilators up in order to accommodate 8 to $12 \mathrm{~F}$ nephrostomy catheters with or without fluoroscopic guidance (see Figure 1, which illustrate stages of insertion of nephrostomy in a patient). Quite often either Locking-Loop Pigtail (LLP) catheters (Cook Inc, USA) or All Purpose Drainage (APD) catheters (Boston Scientic, USA) are used for nephrostomies.

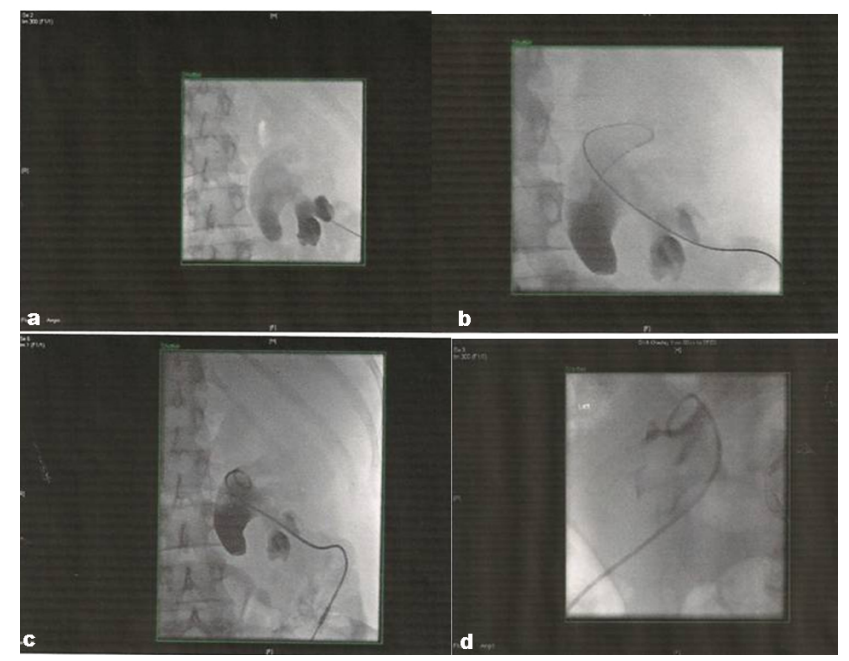

Figure 1: Steps of the procedure of insertion of nephrostomy. a: Right lower calyx was punctured with a Chiba needle. b: Amplatz guide-wire was advanced in to the right renal pelvis. c: Nephrostomy tube size 6 Fr is in situ. d: Left Nephrostomy tube is in situ 
The second technique which is called the "one stab technique" or the "Bonanno technique" entails the use of ultrasound-guided "one-stab" technique using a $6 \mathrm{~F}$ Bonanno catheter (Beckton Dickinson UK Ltd). This $6 \mathrm{~F}$ pigtail Teflon catheter is mounted on a hollow $18 \mathrm{G}$ needle which has a sharp beveled edge. Under guidance of ultrasoundscan the needle tip is inserted and guided into the pelvicalyceal system, upon obtaining urine backflow the catheter is slid over the needle into the collecting system. The nephrostomy catheter is then secured to the skin by means of a catheter fixation disc which is covered with adhesive dressings and connected to a closed urinary drainage bag system.

The ultrasound-guided one-stab technique is a quick and safe procedure in selected cases, and recommended for temporary urinary diversion in cases with moderate to severe degrees of pelvi-calyceal system dilatation. The primary technical success rate is quoted at $88 \%-99 \%$, major complications $4 \%-8 \%$, and minor complications 3\%-15\%. ${ }^{[8]}$ Sonographically guided nephrostomy is the preferred method of treatment in symptomatic renal obstruction or urosepsis during pregnancy. ${ }^{[9]}$

\subsection{Data collection}

The number of patients who had undergone nephrostomy insertions during pregnancy in our hospital over a 5 years period, beginning from January 2009 to end of 2013 was obtained from a list of female patients, who had undergone nephrostomy insertions.

The case notes of the pregnant women who had undergone nephrostomy insertions were used to ascertain information to document their ages, their gestational ages and their symptoms at presentation. The PACS system was used to obtain the following information from the patients' ultrasound (USS) scans, CT scans and nephrostogram images and reports: indications, site of nephrostomy tube insertion(s), gestational ages at which the nephrostomy tubes were inserted and removed, complication(s) of insertion and other treatments given for the complication(s).

The automated letter system (ALS) was used to gather information on clinical history of the patients at presentation and clinical findings on examination, as well as patient outcomes following treatment and subsequent follow-up assessment (including nephrostogram) outcome/treatment. The complications following nephrostomy insertion were noted as well as their management and management outcome.

\section{Results}

A total of 15 patients had nephrostomy over five years.

Two patients presented during the first trimester, 10 patients in the second and three patients in the 3rd trimester respectively of which 14 patients presented with abdominal pain and one patient presented with pain and fever (see Figure 2). USS findings showed that two patients had pyonephrosis, one patient had stone/stricture and 12 patients had hydronephrosis, mild moderate and severe respectively (see Figure 3). Twelve patients had right sided hydronephrosis, one patient had left sided hydronephrosis and two patients had bilateral hydronephrosis (see Figure 4).

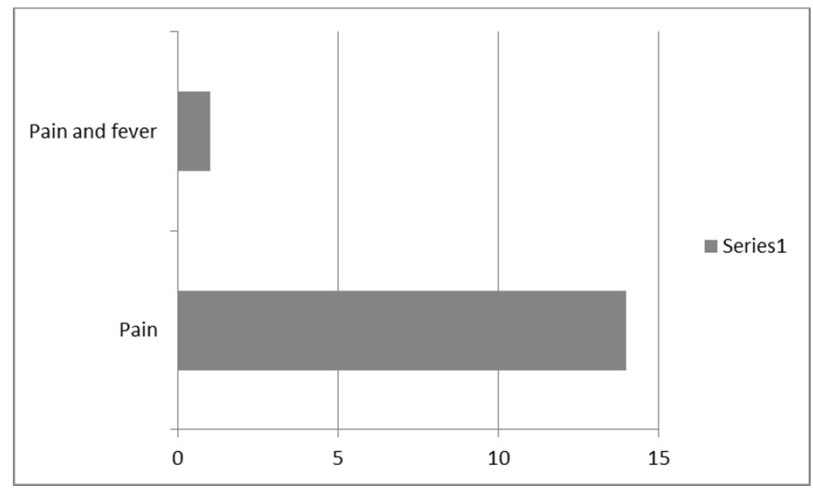

Figure 2: Presentation of the 15 patients

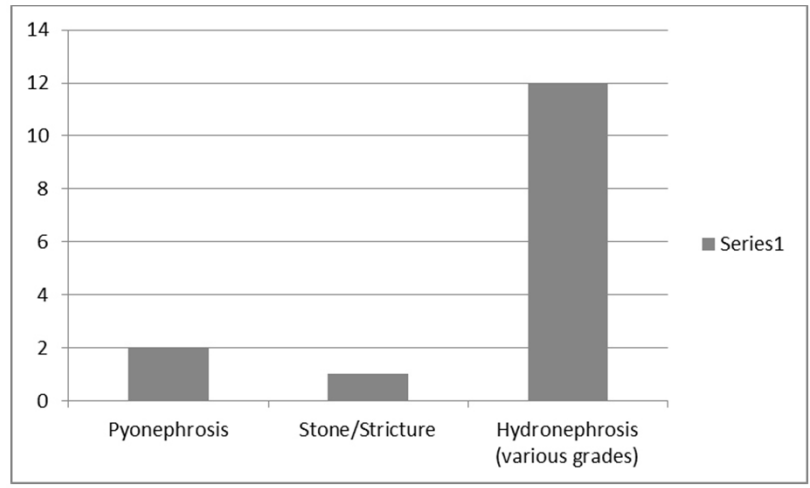

Figure 3: Initial diagnosis based upon ultrasound scan of renal tract

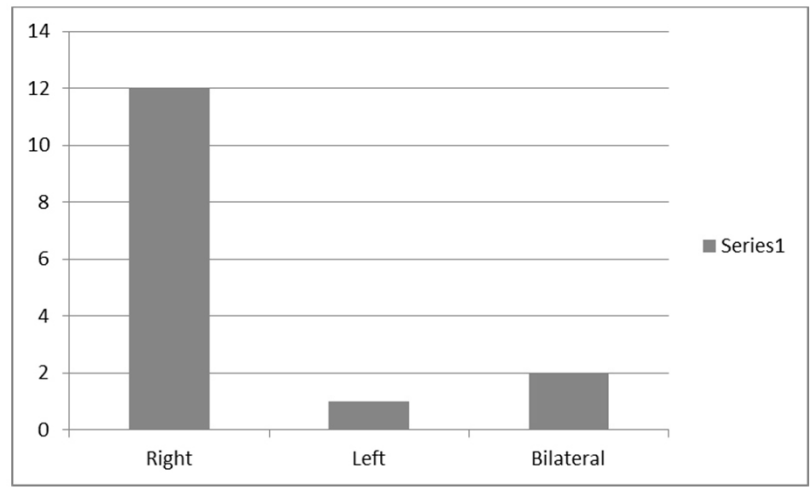

Figure 4: Side of hydronephrosis/nephrostomy tube insertion in the 15 patients

With regard to the timing of the initial insertion of nephrostomy, the results showed that no patient had insertion during 
the first trimester. 11, five, and one patient(s) had insertion during the second and third trimester and post-partum respectively (see Figure 5).

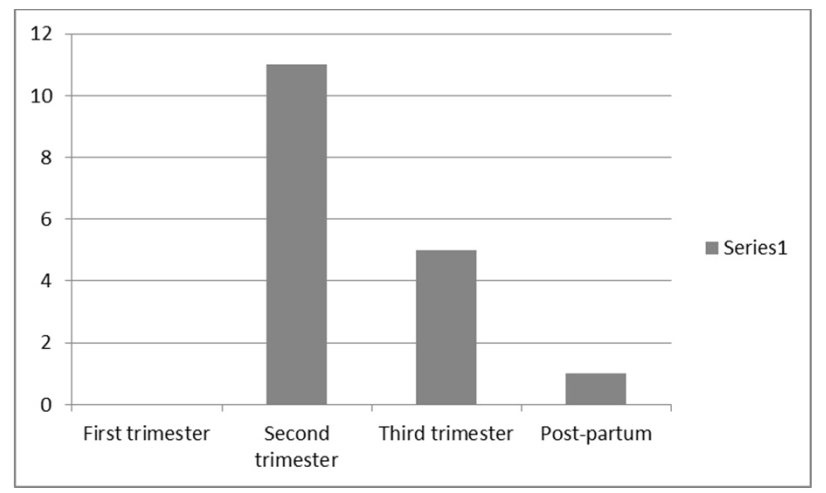

Figure 5: Time of insertion 15 initial nephrostomies plus 2 re-insertions of 2 nephrostomies that fell out

Ten of these patients had blocked nephrostomy tube, 4 had pain from their nephrostomy insertion (pain at nephrostomy site post-insertion) and only one patient was treated for an infection post procedure. Two of the nephrostomy tubes fell out and these were subsequently replaced, a patient developed cyst following insertion and another one had nephrostomy tube calcification and stone formation (see Figure 6).

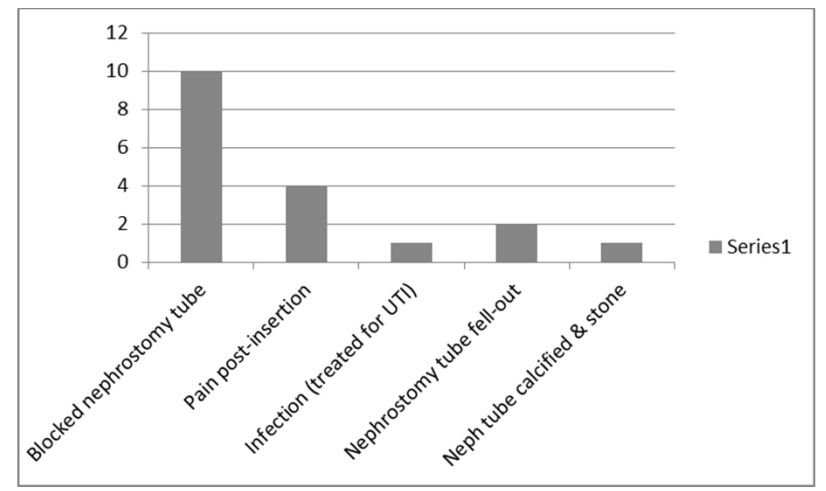

Figure 6: Various complications which emanated during and after insertion of the nephrostomies

Nephrostogram was subsequently done post-partum on eight patients, of which five were normal, two showed right sided stone which was treated post-partum by means of percutaneous nephrolithotomy (PCNL) and one showed both left sided ureteric stricture at the junction of middle and lower one-third of the ureter and right sided stricture at the upper ureter which were treated by balloon dilatation and insertion of JJ ureteric stent (see Figure 7 and 8 which show the stage of gestation at initial presentation of the patients).

\section{Discussion}

In 1991, Schwenke, et al. ${ }^{[10]}$ reported five women who had undergone percutaneous nephrostomy tube insertion over the preceding five years for acute pyelonephritis gravidarum $(n=4)$, among them two with one (single) kidney or asymptomatic collateral excessive ectasia of the pyelo-calyceal system $(n=1)$. The percutaneous nephrostomy insertion was ensued by ante-grade ureteric stent insertion in two cases. Following insertion of the nephrostomies there was a quick clinical but less ultrasound scan evidence of improvement in four patients with improvement in renal function. Delivery was necessary in one case, six days pursuant to the nephrostomy insertion. They also stated that engorged kidneys in pregnancy call for relieving percutaneous nephrostomy if locally delimited or systemic effects threaten to be a risk to the mother and/or the child.

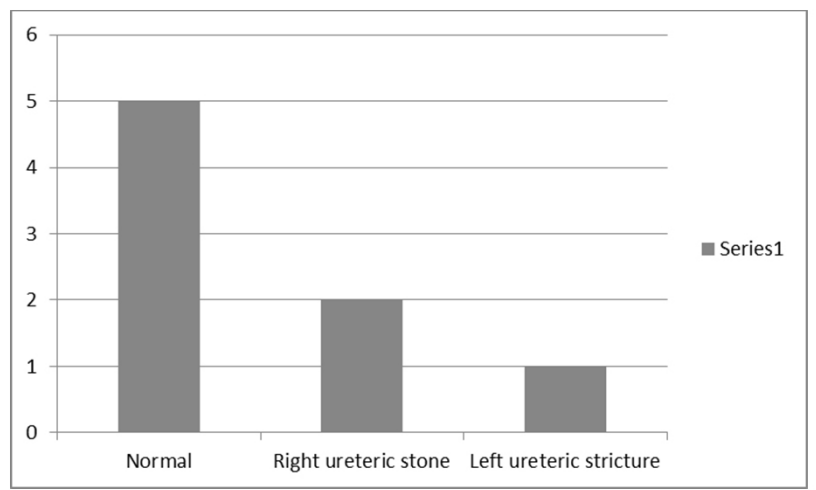

Figure 7: Outcome of nephrostograms performed on eight patients post-partum (remaining seven patients had their nephrostomies removed without nephrostogram with previous evidence of no cause of obstruction on ultrasound scan).

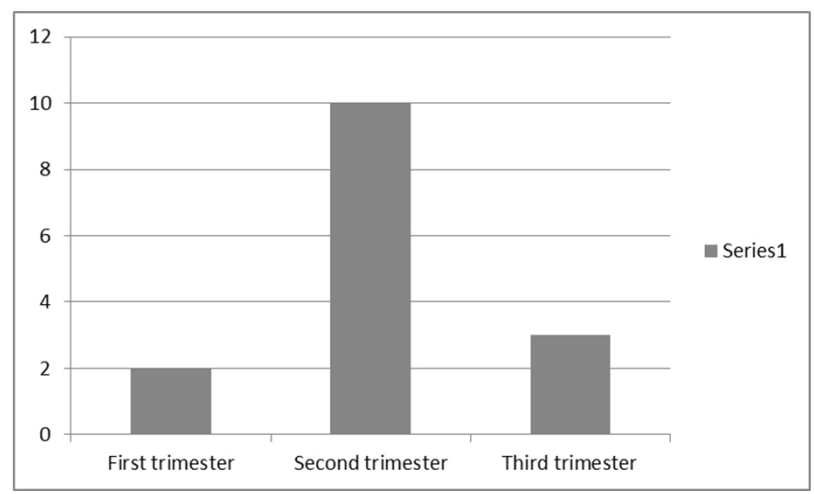

Figure 8: Stage of gestation at initial presentation. Stage of gestation (1st, 2nd, 3rd trimester) at initial presentation.

Semins and Matlaga ${ }^{[11]}$ in 2009 performed a systematic review of MEDLINE and EMBASE from January 1966 to April 2009 in order to obtain information on ureteroscopy 
in pregnancy. They focused on ureteroscopy on pregnant women. They identified a total of 16 publications relating to ureteroscopy in pregnancy in a total of 136 ureteroscopy procedures. With regard to safety of ureteroscopy in pregnancy they reported that out of the 136 procedures involving 136 patients, two had post-operative pain; five had urinary tract infection; there was perforation of ureter in one case; one patient had premature uterine contraction; the remaining 125 patients did not have any complication. Semmins and Matlaga ${ }^{[11]}$ concluded that ureteroscopy is an appropriate intervention in pregnant population with urolithiasis; in all cases ureteroscopy should be performed on a properly selected patient by a surgeon with appropriate experience and equipment. With such an approach, complication rates are low and success rates are high. They emphasized a multidisciplinary approach as a key to successful outcome. They further stated that:

- Urolithiasis during pregnancy is an uncommon but serious problem.

- Options for the treatment of pregnant women with obstructing calculi include: (1) placement of ureteric stent, (2) insertion of percutaneous nephrostomy, and (3) ureteroscopic stone retrieval.

- Even though insertion of a ureteric stent and insertion of percutaneous nephrostomy have been historically the standard treatment of choice for pregnant women with obstructing calculi, there is an emerging collection of literature which had reviewed the safety of ureteroscopy for pregnant women.

Khoo, et al. ${ }^{[12]}$ retrospectively studied the outcome of insertion of percutaneous nephrostomy during pregnancy. They reported that the insertion of percutaneous nephrostomy was successful in all cases $(\mathrm{N}=8,3 \%$ of all nephrostomies over a 5-year period), with one major complication (sepsis) and three minor complications. The nephrostomies remained in situ for a mean of 9.4 days until the stone passed $(n=1)$, or was removed at ureteroscopy $(n=2)$, or a ureteric stent was inserted $(n=5)$. Maternal outcome was normal in all cases but there were two premature deliveries. Khoo, et al. ${ }^{[12]}$ stated that insertion of nephrostomy has an acceptable technical and clinical results but the possibility of an increased risk of septic complications $(12.5 \%$ in their series) would necessitate a further study.

Kavoussi, et al. ${ }^{[13]}$ reported a total of six pregnant women who had obstructing urinary tract calculi which were managed by insertion of PCN under ultrasound-scan guidance and local anesthesia. All of the patients initially had relief of acute obstruction. Nevertheless, occlusion of the nephrostomy tubes by debris necessitating change of nephrostomy occurred in 5 out of the 6 patients. In two patients, as a result of recurrent obstruction, fever and pain percutaneous stone removal during pregnancy was undertaken. The remaining four patients had their nephrostomy tubes in situ until after delivery of their children. During the post-partum period 3 of the patients underwent successful ureteroscopic stone extraction and one patient spontaneously passed the stone. Bacteriuria developed in each patient despite the fact that they were all taking prophylactic antibiotics. All the six patients had successful and uneventful vaginal deliveries with normal healthy babies and the patients themselves remained asymptomatic. Kavoussi, et al. ${ }^{[13]}$ concluded that percutaneous drainage of an acutely obstructed kidney in an obstructed kidney in a pregnant woman is an effective temporizing alternative to insertion of ureteric stent until definite treatment can be performed.

Vansonnenberg, et al. ${ }^{[9]}$ reported their experience in treating seven pregnant women whose ages ranged between 19 years and 31 years who had pyosepsis and dilatation of the upper urinary tract who were successfully treated by means of ultra-sound scan guided percutaneous nephrostomy. The immediate improvement in five patients who were ill because of pyosepsis was dramatic. On the whole 9 percutaneous nephrostomies and 3 tube exchanges were performed in seven pregnant women. Vansonnenberg, et al. ${ }^{[9]}$ stated that prior to their report, earlier reports that focused on noninfected ureteric obstruction during pregnancy had recommended retrograde insertion of ureteric stents and fluoroscopic guided insertion of percutaneous nephrostomy.

In 1988, Quinn, et al. ${ }^{[14]}$ reported a young woman who had markedly obstructed hydronephrosis of pregnancy which had caused forniceal extravasation and this was successfully treated by insertion of PCN.

Peer, et al. ${ }^{[15]}$ reported the use of nephrostomy in four pregnant women, two with ureteric obstruction due to calculi in the ureter, and two with infected hydronephrosis. They stated that:

- $\mathrm{PCN}$ procedure provided rapid relief from pain and pyosepsis and allowed un-eventful continuation of pregnancy to full term and preservation of renal function.

- Traditional methods of urinary diversion in pregnancy included retrograde insertion of ureteric catheters/stents and operative nephrostomy; nevertheless, these techniques required the use of general anesthesia, and were technically difficult and may induce labour.

Jarrard, et al. ${ }^{[16]}$ reported that out of 6,275 pregnancies that were seen in their institution over a two-year period, five patients required operative intervention for acute urinary obstruction unresponsive to medical management. They stated that:

- Ultra-sound scan was able to definitely demonstrate presence of an obstructing calculus in 4 out of 5 patients. 
- With the aid of ultra-sound scan guidance, local anesthesia, and intravenous sedation 7 indwelling ureteric stents were successfully inserted (Ante-grade ureteric stenting procedure is undertaken via a nephrostomy).

- The complication they encountered was distal migration of a ureteric stent in one patient.

- This method of management was successful for symptomatic nephrolithiasis in a pregnant renal transplant patient.

They concluded that:

- Endoscopic placement of ureteric stent under ultrasound scan guidance is an effective and safe method of urinary decompression which is not associated with radiation risks imparted to the mother or fetus and definitive treatment can be deferred to the postpartum period.

- The report of 5 patients out of 6,275 requiring the aforementioned treatment for acute urinary obstruction in pregnancy would indicate a low incidence of about $0.08 \%$ and rarity in pregnant women.

Zwergel, et al. ${ }^{[17]}$ reported the outcome of 116 pregnant women with symptomatic hydronephrosis. They stated that:

- In 30 cases ureteric stents were inserted under local anesthesia. In two cases (pyelo-ureteral stenosis, impacted ureteric stone) percutaneous nephrostomy was necessary.

- Each course of pregnancy and disease was individually decided. In the case of persistent symptoms related to acute hydronephrosis ureteric stent was preferred in view of the fact that it is a simple, safe, and effective method of internal upper urinary tract drainage.

- Acute hydronephrosis during pregnancy which fails to respond to conservative treatment can be managed by ureteric stent insertion or in special cases by insertion of PCN.

Holman, et al. in $1992^{[18]}$ reported that they had removed two pyeloureteric junction stones at 36 weeks and 38 weeks of pregnancy because of complete obstruction and unrelenting spasm. They stated that:

- At that time stone removal was considered more advantageous than PCN alone. This is understandable in that a PCN tract could be dilated at the same time to be used as a portal for removal of the calculi which would avoid longer term placement of nephrostomy tube.

- The patients were placed in semi-oblique prone position with the table raised under the arch of the ribs and a thick hard cushion was placed under the pelvis on the side of the stone. A lead apron was placed under the fetus to reduce the X-ray scatter, and the puncture was made under ultrasound scan guidance. Fetal X-ray exposure was thus reduced to negligible.

- The women delivered baby girls three and five weeks after their percutaneous stone extractions.

- With the necessary skills and carefulness, the intervention is not dangerous for the fetus and that in their opinion it was far more advantageous to perform a definitive - procedure and not to leave a nephrostomy tube in situ for many weeks.

Shah, et al. ${ }^{[19]}$ stated that majority of cases of urolithiasis in pregnancy are managed conservatively either by insertion of ureteric stents or percutaneous nephrostomy which need to be changed at regular intervals and that definitive management of the stone is usually delayed until after delivery of the baby. Shah, et al. ${ }^{[19]}$ reported a patient who presented with pyonephrosis in the 5th week of gestation, due to a stone obstructing the right pelvi-ureteric junction. She was managed by insertion of PCN. She refused to have nephrostomy tube/ureteric stent changes during the rest of her pregnancy and she requested termination of her pregnancy as an alternative to having nephrostomy through-out her pregnancy. She underwent right percutaneous nephrolithotomy in the 14th week of gestation with radiation exposure strictly limited to the kidney for 6 seconds. She delivered a healthy male baby at term.

Fradin, et al. ${ }^{[20]}$ stated that magnetic resonance urographic (MRU) techniques possess image quality and diagnostic capability which are improving with increasingly sophisticated imaging sequences and shorter scanning times. Fradin $^{[20]}$ reported the application of fast-breath hold magnetic resonance (MR) sequence (HASTE) in the assessment of ureteric obstruction in pregnancy. They stated that:

- HASTE MRU was successful in depicting ureteric anatomy and demonstrated bilateral dilatation of the patient's ureters below the pelvic brim. The demonstration suggested distal ureteric obstruction rather than simple hydronephrosis of pregnancy.

- Bilateral nephrostomies were inserted and neonatal prematurity was avoided.

- Furthermore, in their patient HASTE MR Imaging also showed concurrent fetal hydronephrosis.

Rittenberg and Bagley ${ }^{[21]}$ reported the use of flexible ureteroscopy in two pregnant ladies, in one patient the procedure was undertaken for the diagnosis of symptoms simulating ureteric colic, and in the second patient for the removal of a distal ureteric calculus. The diagnostic procedure was completed with local anesthesia alone, and both procedures were carried out without radiation to the fetus or mother. They advocated the early but judicious use of flexible ureteroscopy in pregnant patients with urinary calculi. 
Bozkurt, et al. ${ }^{[22]}$ undertook a retrospective analysis of 32 pregnant women who were referred with hydronephrosis which required surgical treatment. They reported that:

- A 9.5 Fr semi-rigid ureteroscope was used in all cases.

- The ages of the patients ranged from 20 years to 39 years with a mean age of 27.8 years.

- The gestational ages ranged from 15 weeks to 34 weeks (mean 24 weeks). The ultra-sound scan findings were diagnostic of obstructive ureteric calculi in $16(50 \%)$ patients and the mean stone diameter of 8 $\mathrm{mm}$.

- Spinal anesthesia was used in $22(68.8 \%)$ patients, and general anesthesia was used in seven $(21.8 \%)$ patients.

- During endoscopy ureteric calculi were found in 27 $(88.3 \%)$ patients, 10 in distal, nine in mid, and eight in proximal ureter. No stone was found in five patients. The calculi were fragmented with the aid of pneumatic lithotripsy in eight patients and holmium laser was used in 17 patients and the stone fragments were retrieved using forceps.

- With regard to the 32 patients, 19 (59.4\%) had insertion of JJ ureteric stent per-operatively.

- There was no serious intra-operative complication but urinary tract infection in four patients and two patients developed renal colic post-operatively. One patient developed sepsis post-operatively and this was successfully treated with appropriate antibiotics.

- All the babies were born normally.

The authors concluded that semi-rigid ureteroscopy for the diagnosis and treatment of ureteric calculi by intra-corporeal pneumatic or holmium laser lithotripsy is a safe and reasonable option for pregnant women.

Carringer, et al. ${ }^{[23]}$ reported four pregnant women (mean age 29.5 years, range 27-35 years) with 5 episodes of ureteric stones who were treated by means of ureteroscopy and laser lithotripsy when the fetus was at 26 to 35 weeks of gestation. The calculi which were between $5 \mathrm{~mm}$ and 16 $\mathrm{mm}$ in diameter were located in the proximal ureter (1) and distal ureter (4). Carringer, et al. ${ }^{[23]}$ reported that:

- All five calculi were successfully removed by means of ureteroscopy and laser lithotripsy.

- Topical anesthesia was adequate and fluoroscopy was not required in any case.

- They did not observe any complication related to the procedures.

Carringer, et al. ${ }^{[23]}$ concluded that ureteroscopy and laser lithotripsy would appear, in experienced hands, to be a safe and reliable method in the treatment of ureteric calculi during pregnancy. Most cases can be treated without using flu- oroscopy and in some cases the operation could be undertaken under local anesthesia.

Lifshitz and Lingerman ${ }^{[24]}$ reported a retrospective study on 10 consecutive pregnant women who presented with renal colic necessitating intervention. The patients' ages ranged between 17 years and 31 years (mean 23). One of the patients presented during the first trimester, six patients presented during the second trimester and three patients presented during the third trimester. Four of the patients had a past history of stone disease. All of the patients presented with flank pain, with six presenting with left sided pain and four with right sided pain. Haematuria, fever and nausea were present in eight, one, and two patients respectively. With regard to the results, they reported that:

- Ureteroscopy (rigid and/or flexible) was undertaken as a first-line intervention in six patients (in two of them no stone was found).

- Percutaneous nephrolithotomy was undertaken in one patient who presented with a nephrostomy tube already in situ.

- Double J ureteric stents were inserted in three patients for whom the indications were urinary infection, late gestational phase, and difficult ureteroscopy due to narrow ureter respectively.

- They did not observe any urological or obstetric complications. The authors reported the mean size of the stones to be $8 \mathrm{~mm}$.

Lifshitz and Lingerman ${ }^{[24]}$ concluded that ureteroscopy could be considered a safe and effective first-line definitive therapeutic option for pregnant patients requiring intervention for stone disease.

With regard to the incidence of nephrostomy in pregnancy in our trust, over the five year period from beginning of January 2009 to the end of December 2013, there were 49,750 deliveries. The fact that 15 pregnant mothers had nephrostomy within our hospital over the period would indicate that the 15 patients undergoing nephrostomy over the five year period would give an incidence of $0.03 \%$ trust wide. However our trust covers four hospitals and nephrostomy service within our hospital over most of the five year period covered two hospitals in which the total number of deliveries was 24,863 which would make the true incidence of pregnant women undergoing nephrostomy in our catchment area $0.06 \%$. The 15 pregnant women underwent 17 nephrostomies safely and successfully without any immediate problem. There was no significant hemorrhage and no sepsis. There was one episode of minor UTI infection out of the 17 procedures $(5.9 \%)$ which was treated with appropriate oral antibiotics. All the pregnancies continued to full term and all the babies were delivered safely. The nephrostomy tubes were removed post-partum when there was evidence of no obstruction and surgical cause of obstruction was excluded. Nephrostogram undertaken in the post-partum pe- 
riod helped in the establishment of all the surgical causes of obstruction and these were managed appropriately in the post-partum period. The most common problem encountered was blocked nephrostomy tube which required admission of the patients to hospital where the blocked tubes were all successfully flushed to unblock the tubes. The nephrostomy service has a strict protocol which include preprocedure prophylactic antibiotics; normal coagulation, preprocedure analgesia and anti-emetic meditation. Perhaps the low incidence/avoidance of immediate complications, is partly related to the strict adherence to the nephrostomy protocol.

\section{Conclusions}

Out of the numerous patients who have had antenatal and post-natal care in our hospital over a period of five years only 15 had required insertion of percutaneous nephrostomy for various reasons.

The experience gained from the management of our patients would indicate that:

- Majority of these patients would need percutaneous nephrostomy temporarily without any surgical cause found to require subsequent surgical treatment postdelivery and the nephrostomies can safely be removed post-partum.

- Only few patients $3 / 15 \quad(20 \%)$ of the pregnant ladies were found to have surgical causes for their pain and hydronephrosis to require surgical treatment for stones and ureteric strictures (ureteroscopic lithotripsy and dilatation of ureteric strictures).

- Complications/morbidity associated with percutaneous nephrostomy during pregnancy in our experience include: (1) inadequate analgesia or failure to completely avoid pain/discomfort during the procedure of insertion of the nephrostomy; (2) blockage of nephrostomy tube which was a problem in majority of patients 10/15 (about 67\%) and this occurred at home; furthermore the district nurses and general practitioners were unfamiliar with the flushing of the nephrostomies and hence the patients were admitted to hospital and they required the hospital staff in the antenatal wards and at times urologists to flush the nephrostomies to render the nephrostomies patent before the discharge of the patients; (3) infection occurred in one patient (1/15 patients; $1 / 17$ procedures [-5.9\%]) requiring antibiotic treatment; (4) falling out of nephrostomy tube accidentally post-insertion during change of nephrostomy bag which is a known complication occurred in two cases (2/15 patients [13.3\%]) and these required replacement; (5) excessive bleeding requiring blood transfusion and or selective angiography and embolization to stop bleeding which rarely occurs following percutaneous nephros- tomy insertion did not occur in our case.

- It has been the usual practice to avoid CT scan to exclude urinary tract calculi during pregnancy to avoid unnecessary radiation of the baby but occasionally some people would perform a CT scan whilst shielding the baby in utero from the radiation (this approach perhaps would reduce the amount of radiation to the baby but not completely exclude radiation of the baby); occasionally magnetic resonance imaging scan could be used during pregnancy to confirm or exclude the presence of calculus in the urinary tract and to provide reassurance to the expecting mother that there is nothing seriously wrong but the pick-up rate of 3 patients out of $15(20 \%)$ with stones (2) and ureteric strictures is so low that one could wait for a post-partum simple nephrostogram (perhaps magnetic resonance imaging scan could be reserved for cases of previously known urinary tract calculi or predisposing factors to calculi formation like parathyroid tumours and nephrocalcinosis only).

- Safe Ureteroscopic management of ureteric calculi had been occasionally reported in pregnant women and this required the use of general anesthesia; however, this procedure in our opinion should be reserved for selected cases only and routine unrestricted use of ureteroscopy in every pregnant lady who has hydronephrosis in pregnancy would lead to the finding of a normal upper renal tract in most patients which would be adjudged unnecessary procedure requiring general anesthesia in majority of cases.

- Insertion of percutaneous ante-grade ureteric stent is another possibility to avoid long periods of the pregnant lady coping with nephrostomy tubes but this has its disadvantage also in that if the stent is blocked or is associated with obstruction of the ureter a nephrostomy would be required as emergency and that subsequent change of ureteric stent would require cystoscopy which is invasive and would require a general or spinal anesthesia. Furthermore a third of patients with ureteric stents have stent related problems of pain, hematuria, urinary tract infection, and stent migration but nephrostomy change or removal would not require general anesthesia.

\section{Recommendations}

Routine use of ultrasound scan to diagnose increasing hydronephrosis in pregnant ladies with loin pain in addition to full blood count, serum urea and electrolytes, C-reactive protein, bone profile, urinalysis and urine culture should be adequate initial investigation.

Per-cutaneous nephrostomy is a good initial treatment for pain and worsening hydronephrosis, obstructed ureter and pyonephrosis related to pregnancy which should be adequate initial management and this should subsequently be 
followed by post-partum nephrostogram and nephrostomy removal if there is no obstruction or if the upper renal tract is normal and by post-partum treatment of any cause of obstruction like calculus or ureteric stricture.

The use of CT-scan with shielding of the baby from radiation or the use of magnetic resonance imaging scan should be used only in selected occasional cases in which it is adjudged that immediate therapy would be beneficial to the pregnant lady in order to avoid nephrostomy/prolonged stent related symptoms.

Midwives, district nurses and general practitioners should all be taught how to flush nephrostomy tubes so that when a problem of blocked nephrostomy occurs the nephrostomy tube could be flushed at home or in the admitting maternity unit to reduce the rate of readmission of patients with blocked tubes (In this case the authors have found that $2 \mathrm{ml}$ syringe with $2 \mathrm{ml}$ saline flushes blocked nephrostomy tubes better than larger sized syringes).

When there is an endo-urologist and there is evidence of ureteric obstruction due to a calculus occasionally ureteroscopy and endoscopic treatment could be an alternative option of treatment to avoid long periods of nephrostomy in-situ and if required ureteric stents/catheters can in- serted and removed easily within a few days or a week.

\section{Acknowledgements}

Mr. Jason Howard, PACS, Manager of the Radiology Department of North Manchester General Hospital Manchester United Kingdom for getting for us the list of patients who underwent insertion of nephrostomies, United Kingdom; Dr. Emad Bakir, former Consultant Radiologist of North Manchester General Hospital, United Kingdom; Dr. Z Sherazi, consultant radiologist of North Manchester General Hospital, United Kingdom; Dr. E Tam, consultant radiologist of North Manchester General Hospital, United Kingdom; Dr. N Desai, consultant radiologist of North Manchester General Hospital, United Kingdom; Mr. Chris Aitken, Information Manager, Women and Children Division IM and T Services, North Manchester General Hospital, United Kingdom; The Audit Department of North Manchester General Hospital Manchester United Kingdom for making it possible for us to get access to the case notes.

\section{Conflicts of Interest Disclosure}

The authors declare that they do not have conflict of interests.

\section{References}

[1] Gulmi F, Felson D, Vaughan E D. Pathophysiology of urinary tract obstruction. Campbell's Urology: 8th edn. Philadelphia P A Saunders. 2002.

[2] Cheriachan D, Arianayagam M, Rashid P. Symptomatic urinary stone disease in pregnancy. Australian and New Zealand Journal of Obstetrics and Gynaecology. 2008 Feb; 48(1): 34-39. http: //dx.doi.org/10.111/j.1479-828x.2007.00798.x

[3] Stables D. The Renal tract section 2B Pregnancy, the mother. In: Cunningham F G, MacDonald P C, Gant N F, et al. Physiology in Childbearing with Anatomy and Related Biosciences. Edinburgh, UK. Hauscent Publishing Limited. 2000; 236-246.

[4] Cunningham F G, MacDonald P C, Gant N F, et al. Physiology of Pregnancy: Maternal adaptation to pregnancy. In: Cunningham $\mathrm{F}$ G, MacDonald P C, Gant N F, et al, eds. William's Obstetrics, 20th edn. London: Prentice Hall International. 1999; 2191-2365.

[5] Belscher N, Mackay E V, Golditz P B. Medical and Surgical disorders of pregnancy The urinary system. Obstetrics and the Newborn: An illustrated text book, 3rd edn. London: W B Saunders. 1997; 371-376.

[6] Enkin M, Keirse M J, Neillson J P, et al. Infections in Pregnancy. A Guide to Effective Care in pregnancy and Childbirth, 3rd edn, Oxford UK University Press. 2000. http://dx.doi.org/10.1093 /med/9780192631732.001.0001

[7] Lingeman J, Lifshitz D, Am Evan A P. Surgical management of urinary lithiasis. Campbell's urology: 8th edn. Philadelphia, P A Saunders. 2002

[8] Wah T M, Weston M J, Irving H C. Percutaneous nephrostomy insertion: outcome data from prospective multi-operator study at a UK training centre. Clin Radiol. 2004; 59: 255-261. PMid: 15037138. http://dx.doi.org/10.1016/j.crad.2003.10.021
[9] VanSonnenberg E, Casola G, Talner LB, Wittich GR, Varney RR, D'Agostino HB. Symptomatic renal obstruction or urosepsis during pregnancy: treatment by sonographically guided percutaneous nephrostomy. AJR Am J Roentgenol. 1992 Jan; 158(1): 91-94. PMid: 1727366. http://dx.doi.org/10.2214/ajr.158.1.1 727366

[10] Schwenke A I, Fischer W, Schöpke W D. Temporary percutaneous nephrostomy in pregnancy Zentralbi Gynakol. 1991; 113(3): 133139.

[11] Semins M J, Matlaga B R. Ureteroscopy during pregnancy. Indian Journal of Urology. 2009 Jul-Sep; 25(3): 291-295. http: //dx.doi.org/10.4103/0970-1591.56173

[12] Khoo L, Anson K, Patel U. Success and Short-term Complication Rates of Percutaneous Nephrostomy during Pregnancy. Journal of Vascular and Interventional Radiology. 2004 Dec; 15(12): 14691473. PMid: 15590807. http://dx.doi.org/10.1097/01.RV I.0000140639.57131.6D

[13] Kavoussi L R, Abala D M, Basler J W, Able S, Clayman R V. Percutaneous management of urolithiasis during pregnancy. The Journal of Urology. 1992; 148(3 Pt 2): 1069-1071. PMid: 1507334.

[14] Quinn A D, Kusuda L, Amar A D, Das S. Percutaneous nephrostomy for treatment of hydronephrosis of pregnancy. The Journal of Urology. 1988; 139(5): 1037-1038. PMid: 3361638.

[15] Peer A, Strauss S, Witz E, Manor H, Eidelman A. Use of percutaneous nephrostomy in hydronephrosis in pregnancy European Journal of Radiology. 1992 Oct; 15(3): 220-223. http://dx.doi.org /10.1016/0720-048X (92) 90111-L

[16] Jarrad D J, Gerber G S, Lyon E S. Management of acute ureteral obstruction in pregnancy utilizing ultrasound-guided placement of ureteral stents. Urology. 1993 Sep; 42(3): 263-267. http://dx.o $\mathrm{rg} / 10.1016 / 0090-4295$ (93) 90614-G 
[17] Zwergel T, Lindenmeir T, Wullich B. Management of acute hydronephrosis in pregnancy by ureteral stenting. European Urology. 1996; 29(3): 292-297. PMid: 8740034.

[18] Holman E, Tóth C S, Khan M A. Percutaneous Nephrolithotomy in Late Pregnancy. Journal of Endourology. 1992 Dec; 6(6): 421-424. http://dx.doi.org/10.1089/end.1992.6.421

[19] Shah A, Chandak P, Tiptaft R, Glass J, DasGupta P. Percutaneous nephrolithotomy in early pregnancy. The International Journal of Clnical practice (IJCP). 2004 Aug; 58(8): 809-810. http://dx.d oi.org/10.1111/j1368-5031.2004.00047.x

[20] Fradin J M, Regan F, Rodriguez R, Moore R. Hydronephrosis in pregnancy: simultaneous depiction of fetal and maternal hydronephrosis by magnetic resonance urography. Urology. 1999 April; 53(4): 825-827. http://dx.doi.org/10.1016/S0090-4 295 (98) 00411-7
[21] Rittenberg M H, Bagley D H. Ureteroscopic diagnosis and treatment of urinary calculi during pregnancy. Urology. 1988 Nov; 33(5): $427-$ 428. http://dx.doi.org/10.1016/0090-4295(88) 90417-7

[22] Bozkurt Y, Penbegul N, Soylemez H, Atar M, Sancaktutar A A, Yildirim K, et al. The efficacy and safety of ureteroscopy for ureteral calculi in pregnancy: our experience in 32 patients. Urol Res. 2012 Oct; 40(5): 531-535. http://dx.doi.org/10.1007/s00240-0 011-0-454-y

[23] Carringer M, Swartz R, Johansson J E. Management of ureteric calculi during pregnancy by ureteroscopy and laser lithotripsy British Journal of Urology. 1996 Jan; 77(1): 17-20. PMid: 8653305. http: //dx.doi.org/10.1046/j.1464-410X.1996.08433.x

[24] Lifshitz D A, Lingerman J E. Ureteroscopy as a First-Line Intervention for Ureteral Calculi in Pregnancy. Journal of Endourology. 2002 Feb; 16(1): 19-22. http://dx.doi.org/10.1089/08927 7902753483664 